\title{
Precise cosmological parameter estimation using CosmoRec
}

\author{
J. R. Shaw ${ }^{1 \star}$ and J. Chluba ${ }^{1} \dagger$ \\ ${ }^{1}$ Canadian Institute for Theoretical Astrophysics, 60 St. George Street, Toronto, ON M5S 3H8, Canada
}

Received 2011; Accepted 2011

\begin{abstract}
We use the new cosmological recombination code, CosmoRec, for parameter estimation in the context of (future) precise measurements of the CMB temperature and polarization anisotropies. We address the question of how previously neglected physical processes in the recombination model of RECFAST affect the determination of key cosmological parameters, for the first time performing a model-by-model computation of the recombination problem. In particular we ask how the biases depend on different combinations of parameters, e.g. when varying the helium abundance or the effective number of neutrino species in addition to the standard six parameters. We also forecast how important the recombination corrections are for a combined PLANCK, ACTPol and SPTPOL data analysis. Furthermore, we ask which recombination corrections are really crucial for CMB parameter estimation, and whether an approach based on a redshift-dependent correction function to RecFAsT is sufficient in this context.
\end{abstract}

Key words: Cosmic Microwave Background: cosmological recombination, temperature anisotropies, radiative transfer

\section{INTRODUCTION}

The Planck Surveyor ${ }^{1}$ is currently observing the temperature and polarization anisotropies of the cosmic microwave background (CMB) with unprecedented accuracy, constantly producing new exciting results. By now it has successfully completed two full sky scans, and is about to finished its third. On January 11th, 2011, the first release of Planck data became available to the public, e.g. the Early Release Compact Source Catalog (Planck Collaboration et al. 2011a), which also contains the Early Sunyaev-Zel'dovich Cluster Sample (Planck Collaboration et al. 2011b).

Using Planck data, cosmologists will be able to determine the key cosmological parameters with extremely high precision, making it possible to distinguish between various models of inflation (e.g. see Komatsu et al. 2010) by measuring the precise value of the spectral index of scalar perturbations, $n_{\mathrm{S}}$, and constraining its possible running, $n_{\text {run. }}$. In the near future SPTpor ${ }^{2}$ (McMahon et al. 2009 and ACTPor ${ }^{3}$ (Niemack et al. 2010) will provide additional small scale $E$-mode polarization data, complementing the polarization power spectra obtained with Planck .

These encouraging observational prospects have motivated various independent groups (e.g. see Dubrovich \& Grachev 2005 . Chluba \& Sunyaev 2006, Kholupenko \& Ivanchik 2006, Switzer \& Hirata 2008. Wong \& Scott 2007, Rubiño-Martín et al.|2008, Karshenboim \& Ivanov 2008, Hirata 2008, Chluba \& Sunyaev

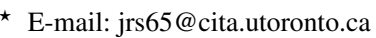

$\dagger$ E-mail: jchluba@cita.utoronto.ca

1 http://www.rssd.esa.int/Planck

2 http://pole.uchicago.edu/

3 http://www.physics.princeton.edu/act/
}

2008, Jentschura 2009, Labzowsky et al. 2009, Grin \& Hirata 2010, Ali-Haïmoud \& Hirata 2010b) to assess how uncertainties in the theoretical treatment of the cosmological recombination process could affect the science return of PLANCK and future CMB experiments. It was shown (Rubiño-Martín et al.2010) that for the standard six parameter cosmology in particular our ability to measure the precise value of $n_{\mathrm{S}}$ and the baryon content of our Universe will be compromised if modifications to the recombination model of RecFast (Seager et al. 1999, 2000) are neglected.

These efforts are now coming to an end, and currently it appears that all important corrections to the standard recombination scenario have been identified (e.g. see Fendt et al. 2009, RubiñoMartín et al.2010, for overview). This has lead to the development of two new independent recombination codes, CosmoRec (Chluba \& Thomas 2010) and HyRec Ali-Haïmoud \& Hirata (2010a), both of which include all important corrections to the recombination problem, superseding the physical model of RecFast. CosmoRec and HyRec allow fast and accurate computations of the ionization history on a model-by-model basis, without the necessity of fudging. A detailed code-comparison is currently in preparation, however, 'out-of-the-box' comparisons indicate agreement at a level of $\sim 0.1 \%-0.2 \%$ during hydrogen recombination $(z \sim 1100)$.

The differences in the free electron fraction with respect to the original version of RECFAST (Seager et al. 1999) reach the level of a $\Delta N_{\mathrm{e}} / N_{\mathrm{e}} \sim 1 \%-3 \%$ (see Fig. 1 for more details). These modifications affect the shape of the Thomson visibility function (Sunyaev \& Zeldovich 1970), slightly shifting its position and changing its width. This leads to important differences in the theoretical predictions of the $\mathrm{CMB}$ temperature and polarisation power spectra, reaching $\Delta C_{l} / C_{l} \sim-4 \%$ at $l \sim 3000$ with respect to the result obtained with the ionisation history given by RecFast (see Fig. 2). 
Neglecting these corrections leads to biases in the inferred parameters, which are important at the level of a few standard deviations for Planck (Rubiño-Martín et al. 2010).

In this paper we address the important question about how the aforementioned modifications to the ionization history propagate into different cosmological parameters. We use the recombination code CosmoRec in connection with CAMB (Lewis et al.2000) and CosmoMC (Lewis \& Bridle 2002) for our computations.

With CosmoRec it has at last become possible to perform model-by-model parameter estimations, including all important physical corrections to the recombination process in a detailed recombination calculation. The study by Rubiño-Martín et al. (2010) was partially reliant on the results of the multi-dimensional interpolation scheme Rico (Fendt et al. 2009), and the final parameter estimations were performed using the newly introduced correction function (Rubiño-Martín et al.2010). Also, at that time the recombination code used for hydrogen (Chluba et al.2007) was limited to 100 shells only, while it is now possible to use effective rates (AliHaïmoud \& Hirata 2010b) for up to 500 shells (Chluba \& Thomas 2010), capturing the correct behaviour in the freeze-out tail of recombination $(z \lesssim 800)$.

Furthermore, we show in more detail how different combinations of parameters affect the associated biases. In particular, in addition to the standard six parameters we allow for a variation of the helium abundance, $Y_{\mathrm{p}}$, and the effective number of neutrino species, $N_{v}$, finding that the main biases are strongly reshuffled (see Table 2 and 3). We also extend the analysis to forecast possible biases to combined Planck plus ACTPol and SPTPol data sets. Finally, we address the question of which physical processes are really important in the recombination correction, and whether an approach based on a correction function (Rubiño-Martín et al. 2010) is sufficient for future parameter estimation.

The paper is structured as follows: in Section 2, we describe CosmoRec and some of the important corrections to the recombination process; in Section 3 we explore the biases produced by different recombination calculations and their implications for cosmological data analysis; finally in Section 4 we discuss the necessary accuracy of calculation required for unbiased analysis of the CMB, providing some details on a RECFAsT correction function approach.

\section{RECOMBINATION PHYSICS}

In this section we briefly discuss the different recombination corrections included in the computations carried out with CosmoRed For a more general overview on recombination physics we refer the interested reader to Fendt et al. (2009), Sunyaev \& Chluba (2009) and Rubiño-Martín et al. (2010).

We study the precision of CosmoRec using different combinations of the recombination physics, as well as various settings for the solver parameters. The main purpose is to demonstrate that using CosmoRec with default setting is sufficient for precise analysis of Planck data, and even for a combination of PlANCK plus ACTPOL and SPTPOL. We also address this question again in Section 3 using CosmoMC. For all results presented in this Section we used the fiducial cosmological model given in Table 2

\footnotetext{
${ }^{4}$ CosmoRec is available at http://www. Chluba.de/CosmoRec
}

\subsection{Corrections to the cosmological ionization history and the CMB power spectra}

CosmoRec allows the incorporation of all known, important, corrections to the cosmological recombination problem. However, its runtime varies rather strongly with the level of detail in the recombination model, and for parameter estimations using CosmoMC it is important to reduce the runtime as far as possible, when using the full recombination calculation. It is therefore useful to check which corrections need to be accounted for to obtain unbiased results in the final parameter estimation.

In Table 1 we define a set of recombination models to illustrate the possible differences in the ionization history. The corresponding corrections to the ionization history as a function of redshift are presented in Fig. 1 and Fig. 2 shows the associated modifications in the CMB power spectra. For the curves given in the left panel of Fig. 1 the output from our $\mathrm{C}++$ version of RecFAs $t^{5}$ was used as reference case, while in the right panel the relative differences between the alternative models are illustrated, with the CosmoRec 'default' case defining the reference.

The left panel of Fig. 1] shows, that the recombination corrections with respect to the original version of RECFAST reach the level of a few percent, both during hydrogen and helium recombination. During helium recombination $(z \sim 1700-2200)$ the largest correction $(\sim-2 \%)$ is because of the increase in the photon escape rate of the $2{ }^{1} \mathrm{P}-1{ }^{1} \mathrm{~S}$ singlet line mediated by the absorption of helium photons in the H I continuum (Kholupenko et al. 2007, Switzer \& Hirata 2008, Rubiño-Martín et al. 2008). During hydrogen recombination $(z \sim 800-1500)$ details in the radiative transfer of the Lyman-series are crucial, including two-photon corrections, resonance scattering, and Raman-events (see Chluba \& Thomas 2010. Ali-Haïmoud \& Hirata 2010b, and references therein for more details). The modification in the CMB power spectra related to the CosmoRec 'default' model are shown in the left panel of Fig. 2

As the right panel of Fig. 1 indicates, the changes in the ionization history between the different models of Table 1 are already very small. For all cases shown the differences in the hydrogen recombination history are $\Delta N_{\mathrm{e}} / N_{\mathrm{e}} \lesssim 0.1 \%$. The largest difference appears when switching off the diffusion correction to the escape probability of the He I $2{ }^{1} \mathrm{P}-1{ }^{1} \mathrm{~S}$ resonance (Rubiño-Martín et al. 2008), resulting in $\Delta N_{\mathrm{e}} / N_{\mathrm{e}} \sim 0.3 \%$ uncertainty at $z \sim 1800$, a correction that currently is not included by HyRec. During hydrogen recombination, higher level two-photon decays $(n>3)$ do still lead to some $\sim 0.1 \%$ uncertainty, which appears to be dominated by the $4 \mathrm{~s}-1 \mathrm{~s}$ and $4 \mathrm{~d}-1 \mathrm{~s}$ process. However, when computing the corresponding changes in the $\mathrm{CMB}$ temperature and polarization power spectra (right panel of Fig 2), it becomes clear that the small difference with respect to the CosmoRec 'default' case will not have a major impact on the cosmological parameter constraints for cosmic variance limited experiments at $l \lesssim 3000$. The agreement in the prediction for the CMB power spectra is better than $0.1 \%$ for all considered recombination models. This error is below the $3 / l$ benchmark suggested by Seljak et al. (2003), and as we will see below, indeed there is no significant bias introduced when choosing between the different recombination models defined in Table 1

These findings suggests that one can use CosmoRec with the

5 RecFast++ is part of CosmoRec and can also be downloaded separately at http://www.Chluba.de/CosmoRec It reproduces the REcFast result (Seager et al. 1999), but avoids any switches in the ODE system (see Fendt et al. 2009 for details). Running RECFAST++ is similar to running RECFAST v1.5 (Wong et al. 2008) with all hydrogen and helium flags set to zero. 

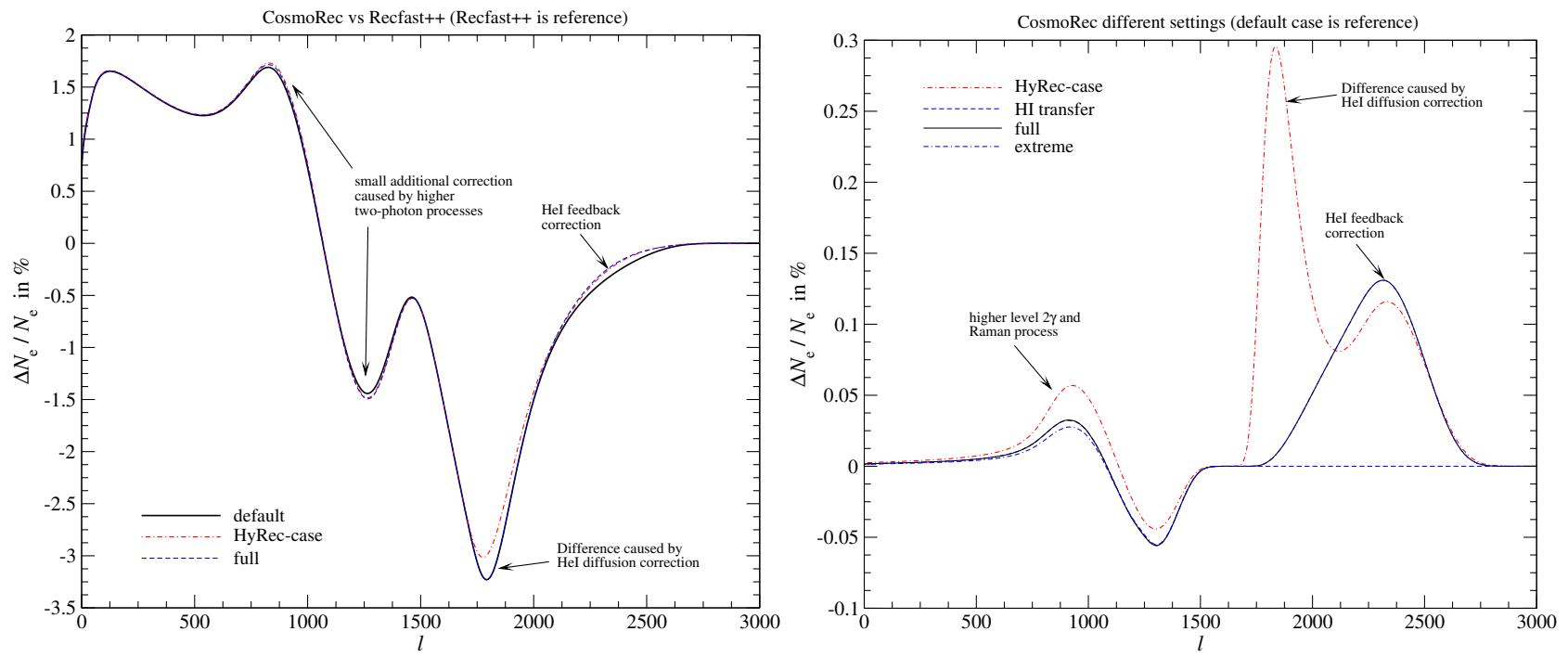

Figure 1. Modifications to the cosmological ionization history. - Left panel: comparison of different recombination models (see Table 1 ) computed using CosmoRec with Recfast++. - Right panel: relative difference between the different recombination models. Here the CosmoRec default case is used as reference.
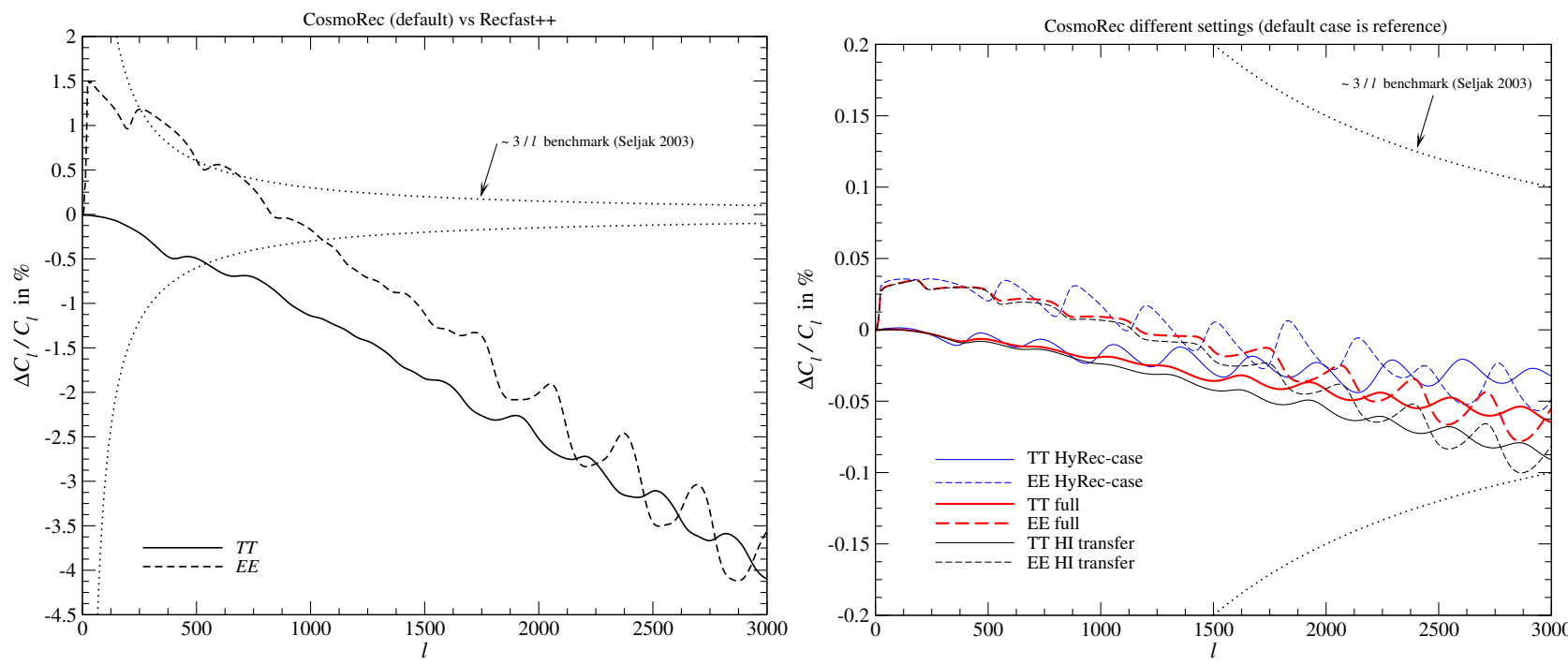

Figure 2. Changes in the CMB temperature and polarization power spectra. - Left panel: correction for the CosmoRec default case with respect to the standard RECFAST++ case. - Right panel: additional corrections for different recombination models. Here the power spectra obtained using the ionization history of the CosmoRec default case define the reference. In both panels we show the $3 / l$ benchmark (Seljak et al. 2003) for comparison (dotted line).

'default' setting to perform accurate cosmological parameter estimations with CosmoMC. Most importantly, for this setting CosMoRec runs in 1.3 seconds per cosmology, and was already tested in a wide range of cosmological parameters to assure stability of the recombination code. In contrast to the most demanding setup ('extreme' case of Table 1) CosmoRec runs about 340 times faster in this mode. We also confirmed the precision of CosmoRec by comparing directly with the most detailed computation carried out using a more elaborate multi-level hydrogen-helium recombination code (Chluba \& Thomas 2010). We found differences no larger than $\Delta N_{\mathrm{e}} / N_{\mathrm{e}} \sim 0.01 \%$ at all redshifts.

We will address the question about the precision of the recombination model more formally in Section 4 however, we find that for accurate parameter estimation using future data from PLANCK , ACTPol and SPTpol the CosmoRec 'default' setting is indeed sufficient. However, we would like to point out that a final cross- validation of the CosmoRec outputs with independent recombination codes (Switzer \& Hirata 2008, Hirata 2008, Grin \& Hirata 2010, Ali-Haïmoud et al. 2010) will be very important. Nevertheless, we do not expect our conclusions to change very much.

\subsection{Modifications to the freeze-out tail of recombination}

Earlier computations of hydrogen recombination (Chluba et al. 2007) were limited to atomic models with $\sim 100$ shells. However, it was later shown that, as expected (Chluba et al.2007), the freezeout tail of recombination $(z \sim 800)$ is still affected significantly until about 300 - 400 shells are included (Grin \& Hirata 2010, Chluba et al.2010). With the effective rate method (Ali-Haïmoud \& Hirata $2010 \mathrm{~b}$ ) it has now become possible to account for this correction in the computation of the CMB power spectra in a very fast way.

When going from 100 shells to 400 shells, the correction in the 
Table 1. Different settings for CosmoRec. In all cases the corrections to the Hi 2s-1s two-photon channel were switched on (Chluba \& Sunyaev 2006 , Kholupenko \& Ivanchik 2006). Furthermore, we used the effective rates for our hydrogen and helium models with $n_{\text {eff }}^{\mathrm{HI}}=500$ and $n_{\text {eff }}^{\mathrm{Hel}}=30$ iChluba \& Sunyaev 2010 Chluba et al. 2010). These were computed with the method of Ali-Haïmoud \& Hirata (2010b). The He I $2^{3} \mathrm{P}-1^{1} \mathrm{~S}$ intercombination line was always switched on (Dubrovich \& Grachev 2005) and the effect of $\mathrm{H}$ I continuum absorption on the $2{ }^{1} \mathrm{P}-1{ }^{1} \mathrm{~S}$ singlet and $2^{3} \mathrm{P}-1{ }^{1} \mathrm{~S}$ triplet resonance (Kholupenko et al.|2007. Switzer \& Hirata 2008) was included using the no redistribution approximation (Rubiño-Martín et al. 2008). In all cases, the detailed history was solved starting at $z=3000$ and ending at $z=50$ with 500 intermediate points. The solution was completed until $z=0$ using the simple RecFAsT ODE system, unless stated differently. Depending on the corresponding settings, the feedback of helium photons was treated according to Chluba \& Sunyaev (2010), including $n^{1} \mathrm{D}-1^{1} \mathrm{~S}$ quadrupole lines and $n^{3} \mathrm{P}-1^{1} \mathrm{~S}$ intercombination lines from levels $n>2$. The diffusion correction to the escape probability of the HEI $2^{1} \mathrm{P}-1{ }^{1} \mathrm{~S}$ resonance was included using the tabulated correction function given in Rubiño-Martín et al. (2008). Higher level two-photon decays and Raman-scatterings were incorporated according to Chluba \& Thomas (2010), giving results that are in excellent agreement with Hirata (2008).

\begin{tabular}{|c|c|c|c|c|c|}
\hline parameter & default & HyRec-case ${ }^{\dagger}$ & HI transfer & full & extreme \\
\hline resolved states & $2 s-3 s, 2 p-3 p, 3 d$ & $2 s-4 s, 2 p-4 p, 3 d-4 d$ & $2 \mathrm{~s}-8 \mathrm{~s}, 2 \mathrm{p}-10 \mathrm{p}, 3 \mathrm{~d}-8 \mathrm{~d}$ & $2 \mathrm{~s}-8 \mathrm{~s}, 2 \mathrm{p}-10 \mathrm{p}, 3 \mathrm{~d}-8 \mathrm{~d}$ & $2 s-8 s, 2 p-10 p, 3 d-8 c$ \\
\hline \# resolved states & 5 & 8 & 22 & 22 & 22 \\
\hline$n_{2 \gamma}$ & 3 & 4 & 8 & 8 & 8 \\
\hline$n_{\text {Raman }}$ & 2 & 3 & 7 & 7 & 7 \\
\hline$n_{\max }^{\mathrm{HeI}}$ & 2 & 2 & 2 & 5 & 5 \\
\hline HeI diffusion correction & on & off & on & on & on \\
\hline HeI feedback & off & $n_{\mathrm{f}}=2$ & off & $n_{\mathrm{f}}=5$ & $n_{\mathrm{f}}=5$ \\
\hline$\Delta z$ (PDE-solver) & 20 & 20 & 2 & 2 & 0.2 \\
\hline average runtime & $1.3 \mathrm{sec}$ & $2.2 \mathrm{sec}$ & $38 \mathrm{sec}$ & $50 \mathrm{sec}$ & $350 \mathrm{sec}$ \\
\hline
\end{tabular}

${ }^{\dagger}$ This case is intended to reproduce the result of HyRec (Ali-Haïmoud \& Hirata 2010a) using CosmoRec.

freeze-out tail of hydrogen changes from about $\Delta N_{\mathrm{e}} / N_{\mathrm{e}} \sim 2.8 \%$ to $\sim 1.6 \%$ (see for example Fig. 2 in Chluba \& Thomas 2010). This is expected to lead to some small change in the CMB power spectra, since the total optical depth to the last scattering surface is slightly modified. However, the difference is very small, and as we show here in detail (Section 4), for precise parameter estimation with Planck it would be sufficient to include only 100 shells to the computation. Although this was already suggested by RubiñoMartín et al. (2010), here we explicitly show this using CosmoRec, however, as the effective rate coefficients can be easily computed even up to 500 shells, this does not lead to any additional obstacle in the recombination calculation.

The main implication of this is that changes at the level of $\sim 1 \%$ in the freeze-out tail of recombination are not constrainable with Planck. This further suggests that modifications caused by collisional processes (Chluba et al. 2007, 2010) at $z \lesssim 800$ should not matter very much. It was already shown that collisions lead to a small acceleration of recombination at $z \lesssim 800$. Since collisional rates are very uncertain, this could imply modifications $\gtrsim 0.1 \%$ (Chluba et al. 2010). However, since changes $\sim 1 \%$ at low redshifts $(z \lesssim 800)$ do not seem to affect the CMB power spectra at a significant level (see Section 4), the above statement appears reasonable. Nevertheless, it will be important to check this with refined computations of collisional rate coefficients.

\subsection{Computation at very low $(z \lesssim 200)$ redshifts}

In the current version of CosmoRec the recombination problem is solved until some lower redshift, $z_{\text {extra }}$. Below this redshift the simple ODE system of RecFast++ is used to complete the solution until $z=0$ (Chluba \& Thomas 2010). The derivatives of CosmoRec at $z_{\text {extra }}$ are used to re-scale the derivatives of RECFAsT ++ accordingly. Clearly this procedure is expected to introduce some small error to the ionization history, however, as Fig. 3 demonstrates the differences are small, when varying the value of $z_{\text {extra }}$. In particular, we

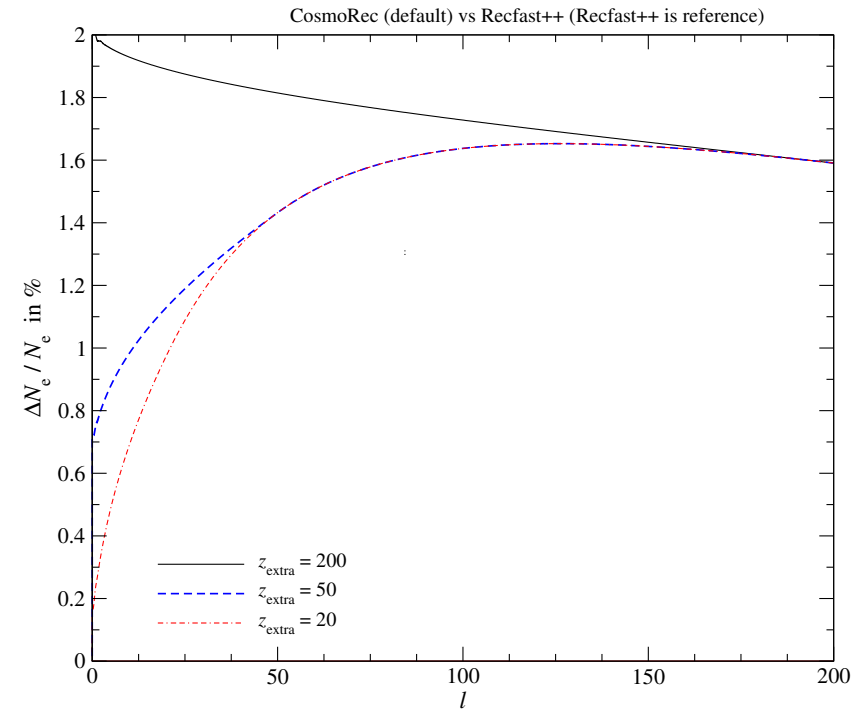

Figure 3. Modifications to the cosmological ionization history at low redshifts. We compare the output of CosmoRec for default setting with RecFAST ++ . The difference in the models is only due to the low- $z$ extrapolation, which is done using the simple RecFast ++ ODE system at $z<z_{\text {extra }}$. The models shown here lead to changes in the CMB power spectra with $\Delta C_{l} / C_{l} \lesssim 10^{-5}$ at $l \lesssim 3000$.

found that the associated uncertainty in the CMB power spectra for all shown cases is $\Delta C_{l} / C_{l} \lesssim 10^{-5}$ at $l \lesssim 3000$. For CosmoRec we shall therefore use $z_{\text {extra }}=50$ for all computations, without introducing any significant change to the CMB power spectra.

This result also suggests that any modifications introduced by details in the primordial chemistry (Stancil et al. 1996, 1998, Schleicher et al. 2008) should not have a major impact on the predictions for the CMB power spectra, as they are expected to be similarly small. Furthermore, at $z \lesssim 10-20$ the process of cosmological 
reionisation (Barkana \& Loeb 2001) after the formation of first stars in the Universe (e.g. see Tegmark et al. 1997; Abel et al. 1997 2000, Yoshida et al. 2007) is expected to occur. This leads to a much larger ambiguity (e.g. see Mortonson \& Hu 2008, for model-independent estimation) in the cosmological ionization history than inherent to detailed recombination calculations. In addition, possible changes caused by energy injection from decaying or annihilating relic particles (e.g. see Chen \& Kamionkowski 2004, Padmanabhan \& Finkbeiner 2005; Zhang et al. 2006 2007, Hütsi et al.2009) and/or cosmic rays (e.g. Jasche et al.2007), could affect the ionization history at these low redshifts. Overall the uncertainty at low redshifts will be dominated by other processes than recombination physics, and detailed analysis of their relevance is beyond the scope of this paper.

\section{PARAMETER ESTIMATION USING COSMOREC}

The difference between ionization history obtained with the original version of RecFast (Seager et al. 1999) and CosmoRed leads to differences in the statistics of the CMB that become increasingly important at small scales (see Section 2). Though these differences are tiny, in any experiment the smallest cosmic variance limited scales are given a huge weight in the likelihood function, and may lead to significant biasing when inferring cosmological parameters. Our task in this Section is to assess the importance of the deviations in recombination calculations on the analysis of future cosmological experiments - our primary approach to this will be to analyse simulated data for various experiments using different recombination calculations and deduce how well the input model is reproduced. We will compare three different recombination calculations CosmoRec, Recfast++ and Recfast++ using a correction function (Rubiño-Martín et al. 2010), which was obtained using the 'full' model given in Table 1 for a cosmology close to the fiducial model (given in Table 2). We will henceforth call RecFast++ with correction function RECFAST ++ CF.

For this work we rely on CosmoMC (Lewis \& Bridle 2002), a sophisticated Markov-Chain Monte-Carlo (MCMC) package that has become the standard code for the analysis of cosmological data. At its core is the Boltzmann code CAMB (Lewis et al.2000) which calculates cosmological statistics given the proposed set of cosmological parameters, and this uses the RECFAST v1.5 system for calculating the baryon ionisation fraction and baryon temperature. At the simplest level, incorporating CosmoRec into the CosmoMC stack is a simple matter of modifying CAMB to replace the RECFAST calculation. We have made these modifications publicly available ${ }^{7}$

In this work we have performed the cosmological analysis using several different models, all based around the standard six parameter $\Lambda \mathrm{CDM}$ model. For parameters we use the dimensionless baryon and dark matter densities, $\Omega_{b} h^{2}$ and $\Omega_{c} h^{2}$; the optical depth to last scattering $\tau$; the spectral index of the primordial power spectrum $n_{s}$ and the logarithm of its amplitude $\log \left(10^{10} A_{s}\right)$ (both defined at a pivot scale of $k_{0}=0.05 \mathrm{Mpc}^{-1}$ ); finally we will use the Hubble parameter $H_{0}$ (instead of $\theta$ ). In choosing this parameterisation we have enforced a flat universe. We are neglecting any secondary contributions to the $\mathrm{CMB}$ - including those from

6 This work was performed using CosmoRec version $1.3 \mathrm{~b}$.

7 The modified code and instructions can be found at http://camb. info/jrs/cosmorec/ the Sunyaev-Zel'dovich effect, gravitational lensing, and inhomogeneous reionisation - as they should be small at the scales we are considering (that is $l<2000$ in all but one case).

The additional models we will consider are all extensions to $\Lambda \mathrm{CDM}$ : allowing the primordial Helium fraction $Y_{\mathrm{p}}$ to vary; having a variable number of neutrino species $N_{v}$ (all of which we assume to be massless); and giving the freedom to fit a more complex primordial power spectrum by adding the running of the spectral index $n_{\text {run }}$ as a parameter. CMB based constraints on $Y_{\mathrm{p}}$ and $N_{v}$ were recently obtained by Dunkley et al. (2010) using combined Aст and WMAP data, demonstrating the power of small scale with full sky experiments. In the future such constraints will tighten very much, and as we show here the recombination corrections with become very important in this case.

For our simulated data we use an exact realisation of the CMB angular powerspectrum calculated for our input model using the all_1_exact functionality of CosmoMc ${ }^{8}$ The noise properties of the simulated data are detailed in subsequent sections. We use no data (simulated or otherwise) in addition to this CMB data. Our input powerspectrum is calculated using the 'full' accuracy setting of CosmoRec (see Table 1) and with all CAMB's accuracy level flags set to 4 . However when performing the analysis we use the 'default' CosmoRec accuracy; we give a detailed discussion of the implications of this choice in Section 4

For analysing cosmic-variance limited data up to a multipole of $l=2000$ the precision of CosmoMC must be increased to at least accuracy_level $=2$. This ensures that it is able to reproduce the simulated data at the precision demanded by the cosmicvariance limit. If the default accuracy of CosmoMC is used, a loglikelihood of $O(1)$ is obtained for the input model (which should have a log-likelihood of zero). In each case discussed we have used at least four chains for the MCMC sampling. We have ensured tested their convergence using the Gelman-Rubin (Gelman \& Rubin 1992) statistic, in all cases terminating when $R-1<0.005$.

\subsection{WMAP}

We have performed an analysis of the WMAP 7-year data release (Komatsu et al.2010) on its own for the models listed above, using the three different recombination calculations. As expected from the work of Rubiño-Martín et al. (2010), there were no observable distinctions between the three, and we confirm that the recombination corrections are not important for WMAP.

\subsection{Parameter estimation for Planck}

Planck is expected to be cosmic variance limited up until $l \sim 1500$ for temperature observations, (though E-mode polarisation is affected by instrumental noise at all scales). This increased precision means it is much more sensitive to the recombination corrections than WMAP (Rubiño-Martín et al. 2010).

We have both repeated the analysis of (Rubiño-Martín et al. 2010), and also extended it by considering models with $Y_{\mathrm{p}}$ and $N_{v}$ included as free parameters. In the simulated data we simply presume that the final Planck map is full sky with the same overall noise properties as the $143 \mathrm{GHz}$ band (assuming the other frequency bands have been used to clean foregrounds). This is the same approximation as Rubiño-Martín et al. (2010). This means we use a

\footnotetext{
${ }^{8}$ See http://cosmocoffee.info/viewtopic. php?t=231
} 
Table 2. The differing recombination calculations could significantly affect the analysis of Planck data. The table below illustrates the biases using each of three different recombination codes, in both their absolute deviation and the number of sigmas. Deviations over $1 \sigma$ are highlighted. For the bias in sigmas we use multiples of the standard deviation of each distribution, a definition that is robust to their non-gaussianity. Though a more desirable alternative would be to turn the confidence limit for each into an effective number of sigmas, this would be highly unreliable as the tails are very sparsely sampled at the large deviations observed.

\begin{tabular}{|c|c|c|c|c|c|c|c|c|}
\hline \multirow[t]{2}{*}{ Parameters } & \multirow[t]{2}{*}{ Fiducial } & \multirow[t]{2}{*}{ Recovered } & \multicolumn{2}{|c|}{ CosmoRec } & \multicolumn{2}{|c|}{ RECFAST++ } & \multicolumn{2}{|c|}{ RECFAST++ w/ correction } \\
\hline & & & Absolute & Sigmas & Absolute & Sigmas & Absolute & Sigmas \\
\hline \multicolumn{9}{|l|}{$\Lambda \mathrm{CDM}$} \\
\hline$\Omega_{b} h^{2}$ & 0.0226 & $0.02260 \pm 0.00014$ & -0.0000 & -0.01 & -0.0003 & -2.10 & 0.0000 & 0.01 \\
\hline$\Omega_{c} h^{2}$ & 0.112 & $0.1121 \pm 0.0012$ & 0.0001 & 0.05 & 0.0006 & 0.48 & 0.0001 & 0.04 \\
\hline$H_{0}$ & 70 & $69.98 \pm 0.60$ & -0.0200 & -0.03 & -0.4800 & -0.78 & -0.0200 & -0.03 \\
\hline$\tau$ & 0.09 & $0.0905 \pm 0.0045$ & 0.0005 & 0.10 & -0.0017 & -0.40 & 0.0003 & 0.07 \\
\hline$n_{s}$ & 0.96 & $0.9598 \pm 0.0036$ & -0.0002 & -0.06 & -0.0120 & -3.35 & -0.0001 & -0.03 \\
\hline $\log \left(10^{10} A_{s}\right)$ & 3.0445 & $3.0456 \pm 0.0089$ & 0.0011 & 0.12 & -0.0091 & -1.05 & 0.0010 & 0.11 \\
\hline \multicolumn{9}{|l|}{$\Lambda \mathrm{CDM}+\mathrm{He}$} \\
\hline$\Omega_{b} h^{2}$ & 0.0226 & $0.02260 \pm 0.00020$ & 0.0000 & 0.02 & $2 \times 10^{-5}$ & 0.10 & $-1 \times 10^{-5}$ & -0.04 \\
\hline$\Omega_{c} h^{2}$ & 0.112 & $0.1120 \pm 0.0013$ & -0.0000 & -0.03 & -0.0002 & -0.17 & 0.0001 & 0.05 \\
\hline$H_{0}$ & 70 & $70.03 \pm 0.72$ & 0.0300 & 0.05 & 0.3800 & 0.51 & -0.0300 & -0.04 \\
\hline$\tau$ & 0.09 & $0.0905 \pm 0.0045$ & 0.0005 & 0.10 & 0.0001 & 0.01 & 0.0003 & 0.06 \\
\hline$n_{s}$ & 0.96 & $0.9601 \pm 0.0069$ & 0.0001 & 0.02 & 0.0004 & 0.05 & -0.0003 & -0.04 \\
\hline $\log \left(10^{10} A_{s}\right)$ & 3.0445 & $3.0454 \pm 0.0095$ & 0.0009 & 0.10 & - 0.0017 & -0.18 & 0.0008 & 0.08 \\
\hline$Y_{p}$ & 0.24 & $0.240 \pm 0.011$ & 0.0000 & 0.01 & 0.0230 & 2.12 & -0.0010 & -0.05 \\
\hline \multicolumn{9}{|c|}{$\Lambda \mathrm{CDM}+$ Neutrinos } \\
\hline$\Omega_{b} h^{2}$ & 0.0226 & $0.02262 \pm 0.00021$ & $2 \times 10^{-5}$ & 0.08 & -0.0000 & -0.02 & $1 \times 10^{-5}$ & 0.04 \\
\hline$\Omega_{c} h^{2}$ & 0.112 & $0.1123 \pm 0.0026$ & 0.0003 & 0.11 & 0.0044 & 1.63 & 0.0001 & 0.05 \\
\hline$H_{0}$ & 70 & $70.2 \pm 1.5$ & 0.2000 & 0.12 & 2.0000 & 1.21 & 0.1000 & 0.05 \\
\hline$\tau$ & 0.09 & $0.0907 \pm 0.0047$ & 0.0007 & 0.14 & -0.0001 & -0.03 & 0.0005 & 0.10 \\
\hline$n_{s}$ & 0.96 & $0.9609 \pm 0.0081$ & 0.0009 & 0.11 & 0.0006 & 0.07 & 0.0004 & 0.05 \\
\hline $\log \left(10^{10} A_{s}\right)$ & 3.0445 & $3.046 \pm 0.012$ & 0.0020 & 0.16 & 0.0040 & 0.33 & 0.0010 & 0.10 \\
\hline$N_{v}$ & 3.046 & $3.07 \pm 0.19$ & 0.0200 & 0.13 & 0.3300 & 1.62 & 0.0100 & 0.05 \\
\hline \multicolumn{9}{|c|}{$\Lambda \mathrm{CDM}+$ Neutrinos $+\mathrm{He}$} \\
\hline$\Omega_{b} h^{2}$ & 0.0226 & $0.02261 \pm 0.00023$ & $1 \times 10^{-5}$ & 0.05 & $5 \times 10^{-5}$ & 0.24 & $1 \times 10^{-5}$ & 0.06 \\
\hline$\Omega_{c} h^{2}$ & 0.112 & $0.1126 \pm 0.0038$ & 0.0006 & 0.17 & 0.0009 & 0.24 & 0.0005 & 0.13 \\
\hline$H_{0}$ & 70 & $70.3 \pm 1.8$ & 0.3000 & 0.18 & 0.9000 & 0.52 & 0.3000 & 0.14 \\
\hline$\tau$ & 0.09 & $0.0905 \pm 0.0046$ & 0.0005 & 0.11 & 0.0001 & 0.03 & 0.0004 & 0.10 \\
\hline$n_{s}$ & 0.96 & $0.9608 \pm 0.0083$ & 0.0008 & 0.10 & 0.0019 & 0.24 & 0.0007 & 0.08 \\
\hline $\log \left(10^{10} A_{s}\right)$ & 3.0445 & $3.046 \pm 0.012$ & 0.0020 & 0.16 & 0.0000 & 0.03 & 0.0020 & 0.13 \\
\hline$N_{v}$ & 3.046 & $3.10 \pm 0.27$ & 0.0500 & 0.19 & 0.0900 & 0.33 & 0.0400 & 0.15 \\
\hline$Y_{p}$ & 0.24 & $0.238 \pm 0.015$ & -0.0020 & -0.13 & 0.0190 & 1.26 & -0.0020 & -0.11 \\
\hline \multicolumn{9}{|c|}{$\Lambda \mathrm{CDM}+$ Running } \\
\hline$\Omega_{b} h^{2}$ & 0.0226 & $0.02260 \pm 0.00015$ & 0.0000 & 0.03 & -0.0002 & -1.40 & 0.0000 & 0.01 \\
\hline$\Omega_{c} h^{2}$ & 0.112 & $0.1119 \pm 0.0013$ & -0.0001 & -0.05 & 0.0006 & 0.45 & 0.0000 & 0.00 \\
\hline$H_{0}$ & 70 & $70.04 \pm 0.62$ & 0.0400 & 0.06 & -0.4100 & -0.65 & 0.0100 & 0.01 \\
\hline$\tau$ & 0.09 & $0.0905 \pm 0.0046$ & 0.0005 & 0.11 & -0.0000 & -0.01 & 0.0004 & 0.08 \\
\hline$n_{s}$ & 0.96 & $0.9601 \pm 0.0036$ & 0.0001 & 0.02 & -0.0121 & -3.42 & 0.0000 & 0.01 \\
\hline $\log \left(10^{10} A_{s}\right)$ & 3.0445 & $3.0454 \pm 0.0096$ & 0.0009 & 0.09 & -0.0043 & -0.44 & 0.0009 & 0.10 \\
\hline$n_{\text {run }}$ & 0 & $-0.0000 \pm 0.0049$ & -0.0000 & -0.01 & -0.0058 & -1.17 & 0.0001 & 0.01 \\
\hline
\end{tabular}

beam scale of $\theta_{\text {beam }}=7.1^{\prime}$, and noise of $\sigma_{T}^{2} \Omega_{\text {beam }}=1.53 \times 10^{-4} \mu \mathrm{K}^{2}$ and $\sigma_{P}^{2} \Omega_{\text {beam }}=5.59 \times 10^{-4} \mu \mathrm{K}^{2}$.

The results of our simulations are summarised in Table 2 For each parameter in the models listed we give its fiducial value, the value constrained by CosmoRec with it standard error, followed by the absolute and relative bias for each of the three recombination calculations. We now discuss specific cases in more detail.

\subsection{1 $\Lambda C D M$ and $\Lambda C D M$ with running}

In the standard $\Lambda \mathrm{CDM}$ case we reproduce the results of RubiñoMartín et al. (2010) finding significant biases in the parameters
$\Omega_{b} h^{2}$ and $n_{s}$ when recovered by RecFast $++(\sim 2 \sigma$ and $3 \sigma$ respectively). From Fig. 2 we can see that the main effect of the recombination corrections on the $C_{l}$ 's is to lower the damping tail further, in light of this it is understandable that the bias obtained shifts $n_{s}$ in order to lower the small scale multipoles. We note that the biases reported here include the total correction to the ionization history with respect to the original version of RECFAST (Seager et al. 1999). In Rubiño-Martín et al. (2010), part of the computations were performed using RecFast v1.4.2, which allows to account for part of the corrections to helium recombination (Wong et al. 2008), using fudge factors. Importantly, in comparison to the original version of RECFAST we find significant shifts for the $\Lambda \mathrm{CDM}+$ running model 
in the parameters $\Omega_{b} h^{2}, n_{s}$ and $n_{\text {run }}$ (shifts of at least $1 \sigma, 3 \sigma$ and $1 \sigma$ respectively), that were not reported in Rubiño-Martín et al.(2010).

\subsection{2 $\Lambda C D M$ with varying $Y_{\mathrm{p}}$ and $N_{v}$}

In the $\Lambda \mathrm{CDM}+$ Helium model we have allowed variation in the primordial Helium fraction $Y_{\mathrm{p}}$ rather than simply relying on the results from Big Bang Nucleosynthesis (see Cyburt et al. 2003. Steigman 2009, for recent constraints and discussion). Running parameter estimations with the RECFAST ++ reveals a large discrepancy in $Y_{\mathrm{p}}$ compared to the fiducial model, recovering a value over $2 \sigma$ higher. As increasing the Helium fraction removes a larger proportion of electrons prior to hydrogen recombination, this change can partially mimic the lower electron fraction found in a full calculation (see Fig. 1). In fact, virtually all other biases disappear when freely varying $Y_{\mathrm{p}}$. With $\sim 0.5 \sigma$ only $H_{0}$ is significantly biased in addition for this case.

The CMB is sensitive to the effective number of neutrino species $N_{v}$ primarily because they affect the background radiation density of the Universe. Any significant deviation from the theoretical value of 3.046 (Mangano et al. 2005) could indicate a further generation of neutrinos, or potentially some other source of background radiation. As illustrated in Table 2, to infer this value correctly requires an accurate calculation of the recombination history. To do otherwise can lead to a bias of up to $1.6 \sigma$ in the recovered value (as shown by using RECFAST++), as well as a similar shift in $\Omega_{b} h^{2}$, and a slightly smaller change in $H_{0}$. The high dimensionality of the parameter space, and the numerous shifts makes it difficult to attribute them to any definite physical effect. We also allow both $Y_{\mathrm{p}}$ and $N_{v}$ as free parameters, in an eight parameter model. This results in a smaller set of shifts, with a higher value of $Y_{\mathrm{p}}$ acquiring the most significant shift (at $1.3 \sigma$ ).

\subsubsection{Parameter estimation using RecFast++CF}

As the results in Table 2 indicate, at the precision of this Planck simulation there is no significant difference between the recovered result for either of the CosmoRec or RECFAST++CF cases for any cosmological model. This confirms that a correction function approach in principle is sufficient for the analysis of PLANCK data, as already pointed out by Rubiño-Martín et al. (2010). However, given that one run of CosmoRec with 'default' setting takes $\sim 1.3$ seconds or about $\sim 15 \%$ of the total CPU time of CAMB per mode 9 it has now become possible to explicitly run the full recombination code, without large penalty.

\subsection{Cosmic variance limited parameter estimation}

Future experiments such as SPTPOL and ACTPol will be much more sensitive than Planck at small scales. This means that the combined Planck plus SPTPOL and ACTPol data sets will be sample variance dominated to much smaller scales than currently accessible ${ }^{10}$ Rather than exploring the consequences of the recombi-

\footnotetext{
9 This is with a timing of 7.5 seconds per CAMB call (at accuracy_level = 2) on a single processor. Using OpENMP, CAMB can be parallelised to reduce its overall time, however, CosmoRec does not benefit from parallelisation at the moment.

${ }^{10}$ Up to $l \sim 2000$ for $E$-mode polarisation in the case of Planck plus the ACTPol wIDE survey. ACTPol DEEP may go as far as $l \sim 4000$. See Niemack et al. 2010
}

nation corrections for any particular experiment, or combination of experiments, we will use a simulated, full-sky, experiment cosmic variance limited until some maximum multipole used in our analysis, usually $l=2000$. Apart from this we perform a similar analysis to the one of the previous Section. Our results are summarised in Table 3

Overall the obtained set of biases for each combination of parameters are similar to the Planck cases shown previously. However, the relative shifts are much greater in every case. For instance in the plain $\Lambda$ CDM model, the bias increases to over 5 and $7 \sigma$ for $\Omega_{b} h^{2}$ and $n_{s}$ respectively (also see Fig. 4). In this case the significant driver of this increase is the overall tightening of the constraints from the added data. However, in many cases there is also an absolute shifting of the biased parameters. This happens as the weight afforded to the largest multipoles eliminates the previously favourable regions, shifting them further away. This is most obvious in the shift of $H_{0}$ in the $\Lambda \mathrm{CDM}+$ Helium model (see Fig. 5 as well as Table 3 .

In Table 3 we also include the results from the $\Lambda \mathrm{CDM}$ model using simulated data from an experiment cosmic variance limited up to $l=3000$. In this case the uncertainty on $\Omega_{b} h^{2}$ reduces by around a half, which increases the associated bias to over $10 \sigma$. For $n_{s}$, however, the distribution width remains comparable, but an increase in the absolute shift, moves the bias to over $12 \sigma$.

Here it is important to mention that at $l \sim 3000$ the additional ambiguities caused by SZ clusters, are expected to mainly impact the $T T$ power spectra, while leaving the $E E$ power spectra less contaminated. This holds the potential that foreground subtraction and the removal of secondary anisotropies up to $l \sim 3000$ could become feasible in the future, in particular for the $E$-mode power spectrum. As our results clearly show, in such cases the recombination corrections will be extremely important.

\section{EFFECT OF PRECISION IN THE RECOMBINATION PHYSICS}

In Section 3 we have used CosmoRec with the 'default' setting to perform model-by-model computations of the recombination process. However, it is clear that this leads to some residual errors with respect to the most precise recombination calculation (model 'extreme' in Table 11. In terms of the CMB power spectra this 'approximation' leads to very small uncertainties, as discussed already in Section 2 (see Fig. 2 for details). Here we ask at what level these differences actually matter for future CMB parameter estimation, using explicit runs of CosmoRec.

\subsection{Changes to the freeze-out tail of recombination}

In Section 2.2 we discussed the effect of the number of shells calculated on the low redshift ionisation fraction. We expect the relative insensitivity of the CMB power spectrum, means that the number of shells included is not of great importance. Using CosmoRec this can be explicitly verified by varying the number of hydrogen levels included into the computation of the effective rate coefficients. In Fig. 6 we can see that even in the cosmic variance limited case there is no significant bias introduced when reducing the number of shells down to 100. For Planck we expect 100 shells to be sufficient, confirming the analysis of Rubiño-Martín et al. (2010). 
Table 3. Deviations between the different recombination calculations for an experiment cosmic-variance limited until $l=2000$ (except in the final case listed). The relative deviations are significantly larger than those in a Planck like experiment (Table 2). Any deviations over $2 \sigma$ have been highlighted.

\begin{tabular}{|c|c|c|c|c|c|c|c|c|}
\hline \multirow[t]{2}{*}{ Parameters } & \multirow[t]{2}{*}{ Fiducial } & \multirow[t]{2}{*}{ Recovered } & \multicolumn{2}{|c|}{ CosmoRec } & \multicolumn{2}{|c|}{ RecFast ++} & \multicolumn{2}{|c|}{ RecFast ++ w/ correction } \\
\hline & & & Absolute & Sigmas & Absolute & Sigmas & Absolute & Sigmas \\
\hline \multicolumn{9}{|l|}{$\Lambda \mathrm{CDM}$} \\
\hline$\Omega_{b} h^{2}$ & 0.0226 & $0.022594 \pm 0.000039$ & $-6 \times 10^{-6}$ & -0.15 & -0.0002 & -5.35 & $-1 \times 10^{-6}$ & -0.01 \\
\hline$\Omega_{c} h^{2}$ & 0.112 & $0.11201 \pm 0.00051$ & $1 \times 10^{-5}$ & 0.01 & -0.0004 & -0.77 & $-2 \times 10^{-5}$ & -0.04 \\
\hline$H_{0}$ & 70 & $69.99 \pm 0.22$ & -0.0100 & -0.03 & 0.0000 & 0.00 & 0.0100 & 0.05 \\
\hline$\tau$ & 0.09 & $0.0901 \pm 0.0020$ & 0.0001 & 0.03 & -0.0013 & -0.64 & 0.0000 & 0.02 \\
\hline$n_{s}$ & 0.96 & $0.9598 \pm 0.0016$ & -0.0002 & -0.14 & -0.0122 & -7.48 & 0.0000 & 0.01 \\
\hline $\log \left(10^{10} A_{s}\right)$ & 3.0445 & $3.0446 \pm 0.0042$ & 0.0001 & 0.04 & -0.0071 & -1.72 & 0.0000 & 0.01 \\
\hline \multicolumn{9}{|l|}{$\Lambda \mathrm{CDM}+\mathrm{He}$} \\
\hline$\Omega_{b} h^{2}$ & 0.0226 & $0.022602 \pm 0.000059$ & $2 \times 10^{-6}$ & 0.03 & 0.0001 & 1.78 & $1 \times 10^{-6}$ & 0.02 \\
\hline$\Omega_{c} h^{2}$ & 0.112 & $0.11201 \pm 0.00051$ & $1 \times 10^{-5}$ & 0.02 & -0.0006 & -1.06 & $6 \times 10^{-5}$ & 0.11 \\
\hline$H_{0}$ & 70 & $70.01 \pm 0.23$ & 0.0100 & 0.03 & 0.6000 & 2.50 & -0.0100 & -0.06 \\
\hline$\tau$ & 0.09 & $0.0901 \pm 0.0021$ & 0.0001 & 0.04 & 0.0002 & 0.08 & 0.0001 & 0.04 \\
\hline$n_{s}$ & 0.96 & $0.9601 \pm 0.0025$ & 0.0001 & 0.03 & 0.0023 & 0.87 & -0.0000 & -0.00 \\
\hline $\log \left(10^{10} A_{s}\right)$ & 3.0445 & $3.0449 \pm 0.0043$ & 0.0004 & 0.10 & -0.0005 & -0.10 & 0.0006 & 0.13 \\
\hline$Y_{p}$ & 0.24 & $0.2405 \pm 0.0035$ & 0.0005 & 0.13 & 0.0250 & 7.24 & 0.0002 & 0.07 \\
\hline \multicolumn{9}{|c|}{$\Lambda \mathrm{CDM}+$ Neutrinos } \\
\hline$\Omega_{b} h^{2}$ & 0.0226 & $0.022597 \pm 0.000058$ & $-3 \times 10^{-6}$ & -0.05 & $5 \times 10^{-5}$ & 0.93 & $-3 \times 10^{-6}$ & -0.06 \\
\hline$\Omega_{c} h^{2}$ & 0.112 & $0.11203 \pm 0.00099$ & $3 \times 10^{-5}$ & 0.03 & 0.0045 & 4.77 & -0.0000 & -0.00 \\
\hline$H_{0}$ & 70 & $69.99 \pm 0.45$ & -0.0100 & -0.02 & 2.2800 & 5.40 & -0.0600 & -0.14 \\
\hline$\tau$ & 0.09 & $0.0902 \pm 0.0021$ & 0.0002 & 0.08 & -0.0002 & -0.11 & 0.0000 & 0.02 \\
\hline$n_{s}$ & 0.96 & $0.9600 \pm 0.0027$ & 0.0000 & 0.01 & 0.0007 & 0.26 & -0.0002 & -0.08 \\
\hline $\log \left(10^{10} A_{s}\right)$ & 3.0445 & $3.0449 \pm 0.0049$ & 0.0004 & 0.08 & 0.0066 & 1.37 & 0.0002 & 0.03 \\
\hline$N_{v}$ & 3.046 & $3.046 \pm 0.058$ & 0.0000 & 0.01 & 0.3460 & 6.35 & -0.0050 & -0.08 \\
\hline \multicolumn{9}{|c|}{$\Lambda \mathrm{CDM}+$ Neutrinos $+\mathrm{He}$} \\
\hline$\Omega_{b} h^{2}$ & 0.0226 & $0.022602 \pm 0.000061$ & $2 \times 10^{-6}$ & 0.04 & 0.0001 & 1.94 & $2 \times 10^{-6}$ & 0.03 \\
\hline$\Omega_{c} h^{2}$ & 0.112 & $0.1121 \pm 0.0014$ & 0.0001 & 0.09 & 0.0007 & 0.50 & 0.0001 & 0.06 \\
\hline$H_{0}$ & 70 & $70.04 \pm 0.54$ & 0.0400 & 0.07 & 1.1000 & 1.94 & 0.0100 & 0.01 \\
\hline$\tau$ & 0.09 & $0.0900 \pm 0.0022$ & 0.0000 & 0.02 & 0.0001 & 0.04 & 0.0001 & 0.04 \\
\hline$n_{s}$ & 0.96 & $0.9601 \pm 0.0028$ & 0.0001 & 0.03 & 0.0032 & 1.11 & -0.0000 & -0.01 \\
\hline $\log \left(10^{10} A_{s}\right)$ & 3.0445 & $3.0448 \pm 0.0051$ & 0.0003 & 0.05 & 0.0016 & 0.31 & 0.0003 & 0.07 \\
\hline$N_{v}$ & 3.046 & $3.054 \pm 0.087$ & 0.0080 & 0.09 & 0.0890 & 1.01 & 0.0040 & 0.04 \\
\hline$Y_{p}$ & 0.24 & $0.2396 \pm 0.0053$ & -0.0004 & -0.07 & 0.0206 & 3.99 & -0.0005 & -0.09 \\
\hline \multicolumn{9}{|c|}{$\Lambda \mathrm{CDM}+$ Running } \\
\hline$\Omega_{b} h^{2}$ & 0.0226 & $0.022594 \pm 0.000043$ & $-6 \times 10^{-6}$ & -0.15 & -0.0002 & -4.05 & $-2 \times 10^{-6}$ & -0.05 \\
\hline$\Omega_{c} h^{2}$ & 0.112 & $0.11203 \pm 0.00055$ & $3 \times 10^{-5}$ & 0.06 & $-7 \times 10^{-5}$ & -0.13 & $4 \times 10^{-5}$ & 0.07 \\
\hline$H_{0}$ & 70 & $69.99 \pm 0.23$ & -0.0100 & -0.06 & -0.1000 & -0.42 & -0.0100 & -0.05 \\
\hline$\tau$ & 0.09 & $0.0901 \pm 0.0022$ & 0.0001 & 0.04 & 0.0006 & 0.28 & 0.0001 & 0.03 \\
\hline$n_{s}$ & 0.96 & $0.9598 \pm 0.0017$ & -0.0002 & -0.10 & -0.0118 & -6.98 & -0.0000 & -0.00 \\
\hline $\log \left(10^{10} A_{s}\right)$ & 3.0445 & $3.0449 \pm 0.0047$ & 0.0004 & 0.08 & -0.0000 & -0.00 & 0.0004 & 0.09 \\
\hline$n_{\text {run }}$ & 0 & $-0.0001 \pm 0.0027$ & -0.0001 & -0.05 & -0.0088 & -3.41 & -0.0001 & -0.03 \\
\hline \multicolumn{9}{|c|}{$\Lambda \mathrm{CDM}\left(\mathrm{CV}\right.$ limited up to $\left.l_{\max }=3000\right)$} \\
\hline$\Omega_{b} h^{2}$ & 0.0226 & $0.022600 \pm 0.000019$ & -0.0000 & -0.03 & -0.0002 & -10.32 & $3 \times 10^{-6}$ & 0.18 \\
\hline$\Omega_{c} h^{2}$ & 0.112 & $0.11202 \pm 0.00047$ & $2 \times 10^{-5}$ & 0.03 & -0.0002 & -0.38 & $4 \times 10^{-5}$ & 0.09 \\
\hline$H_{0}$ & 70 & $69.99 \pm 0.19$ & -0.0100 & -0.03 & -0.1000 & -0.52 & -0.0100 & -0.06 \\
\hline$\tau$ & 0.09 & $0.0901 \pm 0.0020$ & 0.0001 & 0.07 & -0.0021 & -1.18 & 0.0000 & 0.01 \\
\hline$n_{s}$ & 0.96 & $0.9600 \pm 0.0014$ & 0.0000 & 0.03 & -0.0175 & -12.66 & 0.0001 & 0.08 \\
\hline $\log \left(10^{10} A_{s}\right)$ & 3.0445 & $3.0448 \pm 0.0042$ & 0.0003 & 0.08 & -0.0060 & -1.62 & 0.0003 & 0.06 \\
\hline
\end{tabular}

\subsection{CosmoRec 'default' versus 'full' setting}

As remarked upon in the previous section, our simulated data has been generated with the 'full' setting of CosmoRec, which takes around 50 seconds to evaluate the recombination history. However, this is too slow for use during an MCMC analysis, as it would make one execution of CAMB even with highest accuracy setting about seven times slower. By using the 'default' setting we are introducing differences in the power spectrum of around $0.05 \%$ (at $l \sim 2000$; cf. Fig. 2), though small, this induces slight changes to likelihood surface, shifting the preferred parameters. This means that even the CosmoRec case exhibits slight biases when looking at cosmic variance limited data (see Table 3). However, these are limited to around $0.15 \sigma$. This deviation can be reduced by running 

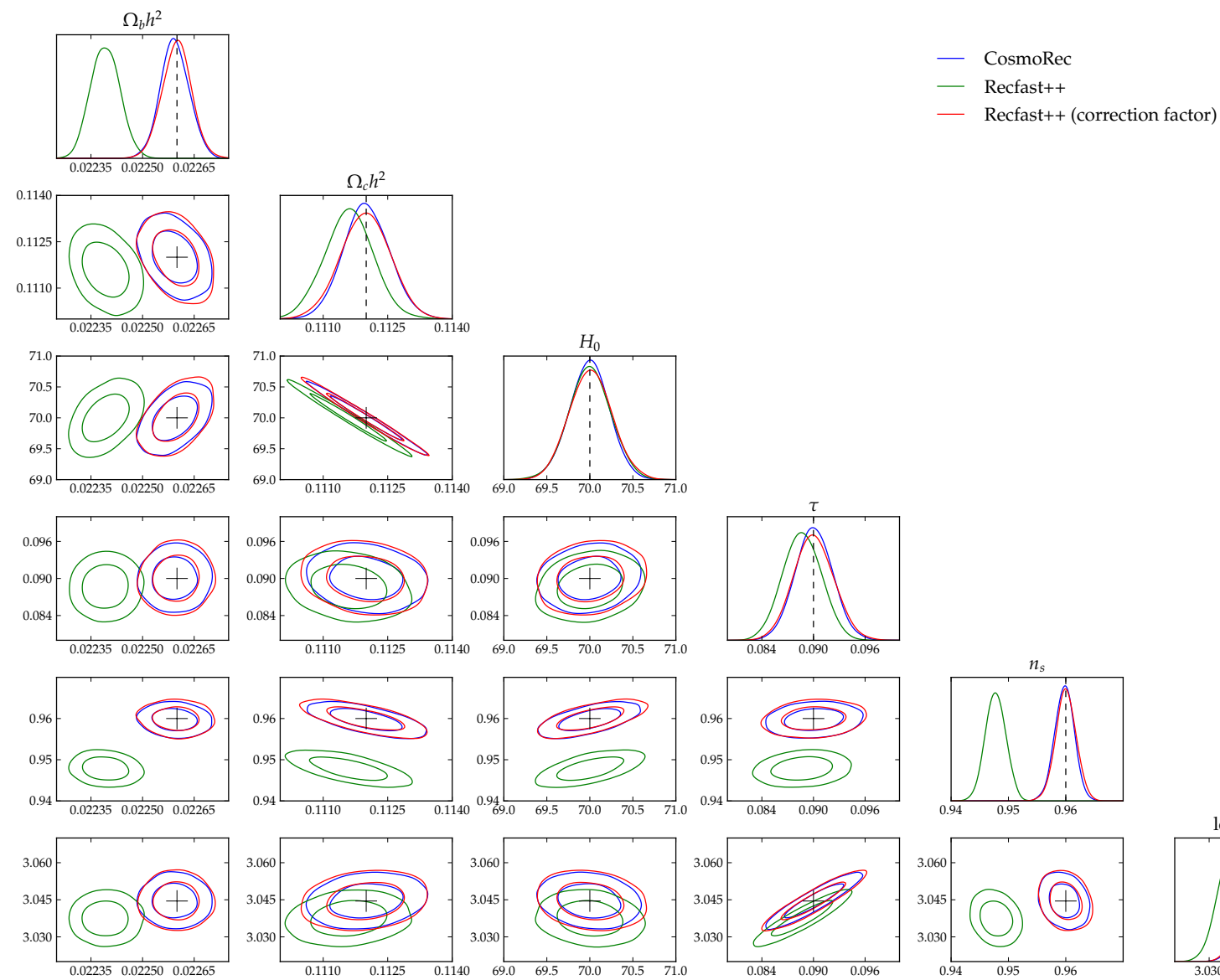

- Recfast++ (correction factor)

Figure 4. Recovered constraints from a simulated experiment, cosmic variance limited in both temperature and polarisation until $l_{\max }=2000$. This plot shows the constraints obtained for the six parameter model, with the dashed lines and crosses marking the input values in the one- and two-dimensional plots. The contours mark the 68\% and 95\% confidence limits of each distribution. The three cases illustrated show that using both CosmoRec and corrected RecFast ++ produce nearly identical probability distributions which faithfully recover the input model. In contrast, the standard RecFasT++ model significantly biases the parameters (most notably $\Omega_{\mathrm{b}} h^{2}$ and $n_{s}$ ).

CosmoRec at higher accuracy. Using the 'HyRec' setting (which does not include the He I diffusion correction, but takes $4 \mathrm{~s} / 4 \mathrm{~d}-1 \mathrm{~s}$ two-photon decay and 3s/3d-1s Raman events into account; see Table 1 for more details) the maximum bias is reduced down to $0.09 \sigma$, and if the He I diffusion corrections is also included this decreases to $0.06 \sigma$. In both cases the runtime of CosmoRec is increased only by about 1 second, a relatively small penalty. We conclude that for Planck parameter analysis the CosmoRec 'default' setting is sufficient, however, if needed additional corrections can be included without compromising the computation time very much. Furthermore, our estimation shows that the He I diffusion correction leads to a small additional modification, which can be safely neglected.

\subsection{Iterative use of RECFAST $++\mathrm{CF}$}

One alternative to using an explicit CosmoRec calculation (or any other more detailed recombination code) is to resort to an incremental approach, based on ReCFAST++ with correction function. In the absence of any prior knowledge about the appropriate cosmology, this would commence with an initial analysis of the data using
RecFast ++ . Then the best fit parameters are used to generate a correction function for that cosmology using the most precise recombination calculation (e.g. Chluba \& Thomas 2010, Ali-Haïmoud \& Hirata 2010a), which should be correct within a nearby region in parameter space. The data is then re-analysed with RecFast++ using this correction function. This process can be iterated until the results are convergent.

We have shown the results of this process in Fig. 7. where we illustrate the $\Omega_{b} h^{2}-n_{s}$ plane (projected from the full $\Lambda$ CDM parameter space), using the cosmic-variance, $l_{\max }=2000$ simulated data. The initial results from RecFast++ are highly biased, as discussed in the previous section. However the first iteration significantly corrects this, with the largest residual being the $0.26 \sigma$ shift in $\Omega_{b} h^{2}$, indicating that 'corrections-to-corrections' are very small already. A further iteration reduces this shift down to only $0.03 \sigma$, suggesting that in this cases two iterations are sufficient. This could likely be reduced down to only one iteration if our cosmological knowledge is sufficient to calculate a suitable initial correction function, eliminating the ReCFast++ only step.

Furthermore, at the initial step one could use ReCFAST v1.5 that 


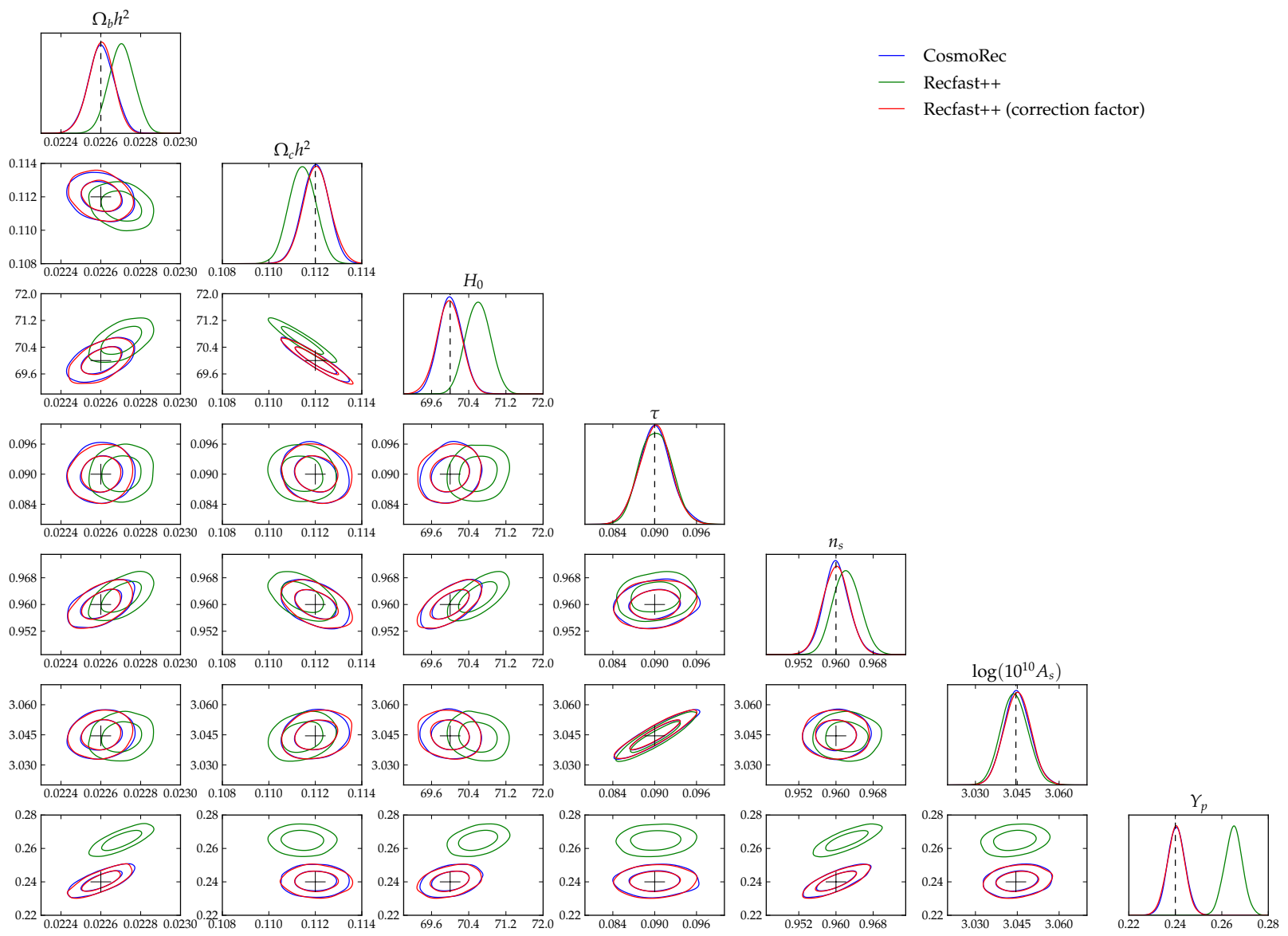

Figure 5. Constraints with our cosmic-variance limited experiment for $l<2000$, for the seven-parameter model of $\Lambda$ CDM plus an unknown primordial Helium content, $Y_{\mathrm{p}}$. The extra degree of freedom shifts the bias of the standard RecFAsT++ calculation into a dramatically higher value of $Y_{\mathrm{p}}$.
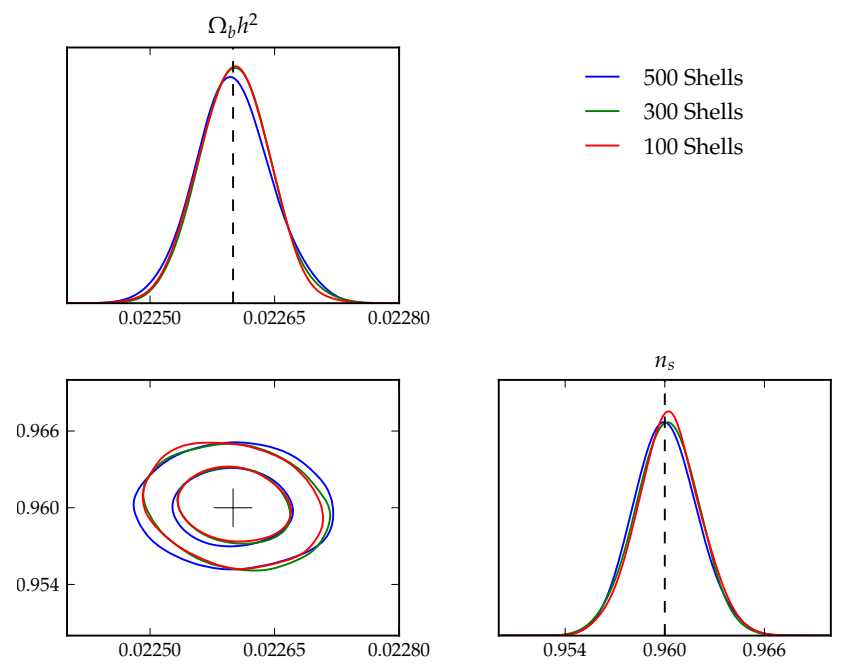

Figure 6. The projection of the $\Omega_{b} h^{2}-n_{s}$ plane from the 6-parameter $\Lambda$ CDM model, analysed using a maximum of 100, 300 and 500 shells in CosmoRec. As can be seen for an experiment cosmic-variance limited at $l=2000$ or less, using a maximum of 100 shells is sufficient. mimics some of the recombination corrections using fudge functions that were calibrated on the results of earlier precise recombination calculations (Rubiño-Martín et al.2010). However, since the fudge function approach (Wong et al.2008) in essence is equivalent to a correction function approach (Rubiño-Martín et al. 2010), one does not expect any additional improvement in this case. Once we are unwilling to run improved recombination codes (i.e. CosmoRec or HyRec), a correction function approach will lead to a very similar approximation at re-calibrated fudge functions to RECFAST.

\section{CONCLUSIONS}

In this work we have demonstrated in detail how previously neglected physical processes during the recombination epoch affect the analysis of precise CMB data from PLANCK and beyond. We have successfully incorporated CosmoRec into the widely used parameter estimation code CosmoMC and the Boltzmann code $\mathrm{CAMB}_{\mathrm{Am}}$ With this we were able to show the parameter biases introduced in a comprehensive set of models when neglecting these corrections.

We confirm the significant biases in the standard six-parameter $\Lambda \mathrm{CDM}$ model previously reported by Rubiño-Martín et al. 2010) for case of PLANCK . In addition to considering a wider range of 

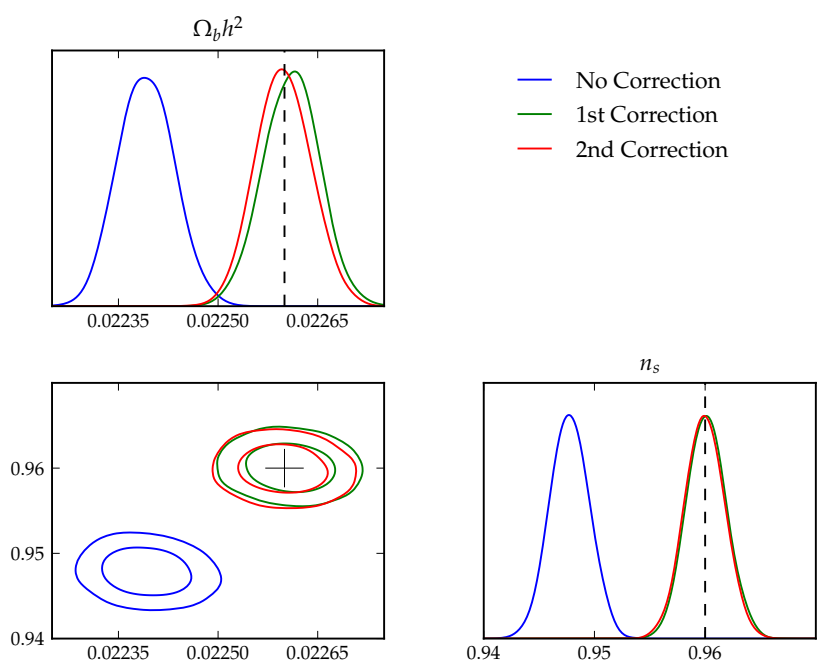

Figure 7. Successive iterations of the REcFast++ correction function in the $\Omega_{b} h^{2}-n_{s}$ plane (taken from a full six-parameter $\Lambda$ CDM analysis). The 'No Correction' plot, calculated using RecFast++, exhibits significant biases, however, from this we can calculate the first correction function. This correction function can be used in ReCFAST++, to produce improved constraints. This iteration is much less biased, with the largest change exhibited a $0.26 \sigma$ shift in $\Omega_{b} h^{2}$. A further iteration with a recalculated correction function, removes the last remaining biases.

cosmological models, we also extend this investigation to a future dataset (comparable to Planck plus ACTPol/SPTpol) using full model-by-model computations of the ionization history with CosMoRec (see Tables 2 and 3 for details). There are several biases of particular note, which have not been mentioned elsewhere:

- Allowing a running of the spectral index yields a bias of over $1 \sigma$ in $n_{\text {run }}$ for Planck. For an experiment that is noise-free up to $l=2000$ this increases to over $3 \sigma$.

- If the primordial Helium fraction is to be inferred using CMB data alone it may be biased by up $7 \sigma$. If uncorrected this would erroneously indicate a significant tension with predictions from BigBang Nucleosynthesis.

- Adding $N_{v}$ as a free parameter would bias its estimated value high by up to $1.6 \sigma$ for PLANCK , however, when also varying the helium fraction this decreases to $0.3 \sigma$ only.

Our analysis indicates that for the current precision of combined WMAP plus ACT data, the bias in $Y_{\mathrm{p}}$ corresponds to about $0.5 \sigma$. This is small compared to the $\sim 2 \sigma$ tension with the BBN value reported by Dunkley et al. (2010). However, in the near future the error in the measurement should decrease and the corresponding bias in $Y_{\mathrm{p}}$ caused by a neglect of detailed recombination physics should exceed the level of $1 \sigma$, even prior to the final release of Planck data.

Although small scale CMB data is able to internally break many of the degeneracies of current cosmological analyses, it will still be desirable to supplement future CMB data with complementary probes such as Type Ia supernovae data (Amanullah et al. 2010) or BAO measurements (Schlegel et al. 2009). We have not included any additional data in this work, and whilst it may serve to reduce the overall biases obtained (if using a RECFAsT analysis), the tension between datasets is likely to artificially tighten parameter constraints.

In order to focus on the importance of the recombination corrections we have necessarily neglected many instrumental and physical systematics:

- Both the SZ effect and gravitational lensing will significantly add to the small scale CMB. We have neglected these in our analysis assuming that for $l<2000$ they will be negligible or sufficiently well understood.

- Our work uses a simple reionisation model which is assumed to be known (other than a single parameter giving the optical depth). A more complicated homogeneous reionisation history should only affect the large scale multipoles, whereas any realistic inhomogeneity will not significantly effect multipoles $l \sim 2000$ (see e.g., Zahn et al.|2005).

- We have assumed a simple, perfectly known, noise model in this work. In reality beam, gain and other uncertainties will have to be considered (see e.g. Colombo et al. 2009).

The 'default' accuracy setting for CosmoRec is designed to be a fast enough to be used for MCMC analysis of cosmological data, and this work shows that it is sufficiently accurate for the unbiased analysis of PLANCK data. We also discuss an iterative approach using RecFast++ with a correction function. Though this approach is accurate (see Sect. 4.3), it is simpler and quicker overall to directly use the full CosmoRec analysis. For the analysis of future Planck plus ACTPol/SPTpol data, we show that CosmoRec with 'default' setting is unbiased at the $0.15 \sigma$ level, which can be significantly reduced by running at a higher accuracy setting (leading to a small runtime increase).

It is important to note that a final cross-validation of the CosMoREc outputs with independent recombination codes (Switzer \& Hirata 2008, Hirata 2008, Grin \& Hirata 2010, Ali-Haïmoud et al. 2010) will be very important, for confirming their veracity. Nevertheless, initial comparisons mean that we do not expect any significant changes (at least for the experiments simulated in this work).

We would also like to mention that in this work we have demonstrated the importance of the total corrections to the ionization history with respect to the original RECFAst code (Seager et al. 1999). This correction was obtained in a common effort by several independent groups, and part of these corrections are now included by the latest version of RecFAst using calibrated correction functions, based on the more detailed recombination computations. As we demonstrated here, such an approach is similar to an iterative scheme using a precomputed correction function for the original RecFast computation (see Sect.4.3. However, we also argued that for runtimes achieved with recent recombination codes, such an approximate approach can be avoided.

\section{ACKNOWLEDGMENTS}

The authors would like to thank Yacine Ali-Haïmoud, Dick Bond, Dan Grin, Amir Hajian, Chris Hirata, Antony Lewis, Jose-Alberto Rubiño-Martín, Mike Nolta, Hiranya Peiris, Douglas Scott, Rashid Sunyaev and Eric Switzer for useful and stimulating discussions of the problem. JC is also very grateful for additional financial support from the Beatrice D. Tremaine fellowship 2010. Furthermore, we acknowledge the use of the GPC supercomputer at the SciNet HPC Consortium. SciNet is funded by: the Canada Foundation for Innovation under the auspices of Compute Canada; the Government of Ontario; Ontario Research Fund - Research Excellence; and the University of Toronto. 


\section{REFERENCES}

Abel T., Anninos P., Zhang Y., Norman M. L., 1997, New Astronomy, 2, 181

Abel T., Bryan G. L., Norman M. L., 2000, ApJ, 540, 39

Ali-Haïmoud Y., Grin D., Hirata C. M., 2010, ArXiv e-prints

Ali-Haïmoud Y., Hirata C. M., 2010a, ArXiv e-prints

Ali-Haïmoud Y., Hirata C. M., 2010b, Phys.Rev.D, 82, 063521

Amanullah R., Lidman C., Rubin D., Aldering G., Astier P., Barbary K., Burns M. S., Conley A., Dawson K. S., Deustua S. E., Doi M., Fabbro S., Faccioli L., Fakhouri H. K., Folatelli G., Supernova Cosmology Project T., 2010, ApJ, 716, 712

Barkana R., Loeb A., 2001, Physics Reports, 349, 125

Chen X., Kamionkowski M., 2004, Phys.Rev.D, 70, 043502

Chluba J., Rubiño-Martín J. A., Sunyaev R. A., 2007, MNRAS, 374,1310

Chluba J., Sunyaev R. A., 2006, A\&A, 446, 39

Chluba J., Sunyaev R. A., 2008, A\&A, 480, 629

Chluba J., Sunyaev R. A., 2010, MNRAS, 402, 1221

Chluba J., Thomas R. M., 2010, ArXiv e-prints

Chluba J., Vasil G. M., Dursi L. J., 2010, MNRAS, 407, 599

Colombo L. P. L., Pierpaoli E., Pritchard J. R., 2009, MNRAS, 398, 1621

Cyburt R. H., Fields B. D., Olive K. A., 2003, Physics Letters B, 567, 227

Dubrovich V. K., Grachev S. I., 2005, Astronomy Letters, 31, 359

Dunkley J., Hlozek R., Sievers J., Acquaviva V., Ade P. A. R., Aguirre P., Amiri M., Appel J. W., Barrientos L. F., Battistelli E. S., Bond J. R., 2010, ArXiv e-prints

Fendt W. A., Chluba J., Rubiño-Martín J. A., Wandelt B. D., 2009, ApJS, 181, 627

Gelman A., Rubin D., 1992, Statistical science, 7, 457

Grin D., Hirata C. M., 2010, Phys.Rev.D, 81, 083005

Hirata C. M., 2008, Phys.Rev.D, 78, 023001

Hütsi G., Hektor A., Raidal M., 2009, A\&A, 505, 999

Jasche J., Ciardi B., Enßlin T. A., 2007, MNRAS, 380, 417

Jentschura U. D., 2009, Phys.Rev.A, 79, 022510

Karshenboim S. G., Ivanov V. G., 2008, Astronomy Letters, 34, 289

Kholupenko E. E., Ivanchik A. V., 2006, Astronomy Letters, 32, 795

Kholupenko E. E., Ivanchik A. V., Varshalovich D. A., 2007, MNRAS, 378, L39

Komatsu E., Smith K. M., Dunkley J., Bennett C. L., Gold B., Hinshaw G., Jarosik N., Larson D., Nolta M. R., Page L., Spergel D. N., Halpern M., 2010, ArXiv e-prints

Labzowsky L., Solovyev D., Plunien G., 2009, Phys.Rev.A, 80, 062514

Lewis A., Bridle S., 2002, Phys. Rev., D66, 103511

Lewis A., Challinor A., Lasenby A., 2000, ApJ, 538, 473

Mangano G., Miele G., Pastor S., Pinto T., Pisanti O., Serpico P. D., 2005, Nuclear Physics B, 729, 221

McMahon J. J., Aird K. A., Benson B. A., Bleem L. E., Britton J., Carlstrom J. E., Chang C. L., Cho H. S., de Haan T., Crawford T. M., Crites A. T. a., 2009, in B. Young, B. Cabrera, \& A. Miller ed., American Institute of Physics Conference Series Vol. 1185 of American Institute of Physics Conference Series, SPTpol: an instrument for CMB polarization. pp 511-514

Mortonson M. J., Hu W., 2008, ApJ, 672, 737

Niemack M. D., Ade P. A. R., Aguirre J., Barrientos F., Beall J. A., Bond J. R., Britton J., Cho H. M., Das S., Devlin M. J., Dicker S., Dunkley J., 2010, in Society of Photo-Optical Instrumentation
Engineers (SPIE) Conference Series Vol. 7741 of Presented at the Society of Photo-Optical Instrumentation Engineers (SPIE) Conference, ACTPol: a polarization-sensitive receiver for the Atacama Cosmology Telescope

Padmanabhan N., Finkbeiner D. P., 2005, Phys.Rev.D, 72, 023508

Planck Collaboration Ade P. A. R., Aghanim N., Arnaud M., Ashdown M., Aumont J., Baccigalupi C., Balbi A., Banday A. J., Barreiro R. B., et al. 2011b, ArXiv e-prints

Planck Collaboration Ade P. A. R., Aghanim N., Arnaud M., Ashdown M., Aumont J., Baccigalupi C., Balbi A., Banday A. J., Barreiro R. B., et al. 2011a, ArXiv e-prints

Rubiño-Martín J. A., Chluba J., Fendt W. A., Wandelt B. D., 2010, MNRAS, 403, 439

Rubiño-Martín J. A., Chluba J., Sunyaev R. A., 2008, A\&A, 485, 377

Schlegel D. J., Bebek C., Heetderks H., Ho S., Lampton M., Levi M., Mostek N., Padmanabhan N., Perlmutter S., Roe N., Sholl M., Smoot G., 2009, ArXiv e-prints

Schleicher D. R. G., Galli D., Palla F., Camenzind M., Klessen

R. S., Bartelmann M., Glover S. C. O., 2008, A\&A, 490, 521

Seager S., Sasselov D. D., Scott D., 1999, ApJL, 523, L1

Seager S., Sasselov D. D., Scott D., 2000, ApJS, 128, 407

Seljak U., Sugiyama N., White M., Zaldarriaga M., 2003, Phys.Rev.D, 68, 083507

Stancil P. C., Lepp S., Dalgarno A., 1996, ApJ, 458, 401

Stancil P. C., Lepp S., Dalgarno A., 1998, The Astrophysical Journal, 509, 1

Steigman G., 2009, arXiv, astro-ph.GA

Sunyaev R. A., Chluba J., 2009, Astronomische Nachrichten, 330, 657

Sunyaev R. A., Zeldovich Y. B., 1970, Astrophysics and Space Science, 7, 3

Switzer E. R., Hirata C. M., 2008, Phys.Rev.D, 77, 083006

Tegmark M., Silk J., Rees M. J., Blanchard A., Abel T., Palla F., 1997, ApJ, 474, 1

Wong W. Y., Moss A., Scott D., 2008, MNRAS, 386, 1023

Wong W. Y., Scott D., 2007, MNRAS, 375, 1441

Yoshida N., Omukai K., Hernquist L., 2007, The Astrophysical Journal, 667, L117

Zahn O., Zaldarriaga M., Hernquist L., McQuinn M., 2005, ApJ, 630, 657

Zhang L., Chen X., Kamionkowski M., Si Z., Zheng Z., 2007, Phys.Rev.D, 76, 061301

Zhang L., Chen X., Lei Y., Si Z., 2006, Phys.Rev.D, 74, 103519 